\title{
Clinical Characteristics of Respiratory Tract-Associated Streptococcus pyogenes at General Japanese Hospital in 2014
}

\author{
Masaaki Minami ${ }^{*}$, Ryoko Sakakibara², Taichi Imura², Mika Watanabe ${ }^{2}$, Hideo Morita², \\ Naoto Kanemaki ${ }^{3}$, Michio Ohta ${ }^{4}$ \\ ${ }^{1}$ Department of Bacteriology, Graduate School of Medical Sciences, Nagoya City University, Nagoya, Japan \\ ${ }^{2}$ Department of Clinical Investigation, Daido Hospital, Nagoya, Japan \\ ${ }^{3}$ Department of Gastroenterology, Daido Hospital, Nagoya, Japan \\ ${ }^{4}$ School of Nursing, Sugiyama Jyogakuen University, Nagoya, Japan \\ Email: *minami@med.nagoya-cu.ac.jp
}

Received 15 October 2015; accepted 11 December 2015; published 18 December 2015

\begin{abstract}
Streptococcus pyogenesis most popularpathogen caused respiratory tract infection disease. This study was conducted to find out the recent clinical characteristics of Streptococcus pyogenes isolates from respiratory tract at Japanese hospital in 2014. Streptococcus pyogenes was identified by standard laboratory procedure. Antimicrobial susceptibility testing was performed by micro dilution assay according to CLSI recommendation. Respiratory tract isolates were defined as isolation from pharynx, nasal discharge, tonsillar, and sputum. Total one hundred sixty-one Streptococcus pyogenes were isolated among which 136 were from respiratory tract and 25 were from non- respiratory tract. Respiratory tract-associated Streptococcus pyogenes was isolated among which 102 were from male and 59 were from female. The age incidence among 0 - 1 years age group was 8 , among 1 - 18 years age group, $131(p<0.01)$, among 19 - 64 years age group, $17(p<0.01)$, and in 65 -years it was 5 . Although we investigated the relationship between season and bacterial isolation, we did not find any significant differences between respiratory tract and no respiratory tract isolation. However, our study revealed that clarithromycin was less antimicrobial effective in respiratory tract disease than in no respiratory tract disease significantly $(p<0.01)$. Although several antibiotics such as penicillin are still effective against Streptococcus pyogenes, incidence of Streptococcus pyogenes infection is not decreasing worldwide. Our study suggests the need for continuous epidemiological surveillance of Streptococcus pyogenes.
\end{abstract}

\section{Keywords}

Streptococcus pyogenes, Susceptibility, Antimicrobial Resistance, Epidemiology, Clarithromycin

\section{Introduction}

Streptococcus pyogenes (Lancefield group A) is a human pathogen responsible for a wide range of diseases, the ${ }^{*}$ Corresponding author.

How to cite this paper: Minami, M., Sakakibara, R., Imura, T., Watanabe, M., Morita, H., Kanemaki, N. and Ohta, M. (2015) Clinical Characteristics of Respiratory Tract-Associated Streptococcus pyogenes at General Japanese Hospital in 2014. Journal of Biosciences and Medicines, 3, 26-31. http://dx.doi.org/10.4236/jbm.2015.312005 
most common of which are upper respiratory tract (nasopharyngeal) infections and impetigo [1].

Streptococcus pyogenes infection has been described since the time of Hippocrates. In the $16^{\text {th }}$ century, severe forms of pharyngitis occurring in Spain were referred to as "garitillo" and in England in the $18^{\text {th }}$ century were known as "Fothergill's sore throat" [2]. Although one strain may emerge as a dominant strain, only to be replaced by one or more different strains over a 10-year period, the annual prevalence of Streptococcal pharyngitis has remained quite constant over decades [3]. Pharyngitis may progress to scarlet fever, bacteremia, suppurative head and neck infections, rheumatic fever, post streptococcal glomerulonephritis, or streptococcal toxic shock syndrome [2]. Streptococcus pyogenes may be isolated from the throats of $1 \%$ to $70 \%$ of the population, many of whom are asymptomatic [4]. Patients with pharyngitis have abrupt onset of sore throat, submandibular adenopathy, fever, and chilliness [2] [4].

Streptococcus pyogenes is even more remarkable in terms of the very large number of factors that have been identified as potential virulence determinants for those various disease [5]. These include surface proteins ( $\mathrm{M}$ proteins, fibronectin-binding proteins, surface dehydrogenases, C5a peptidase), the hyaluronic acid capsule, secreted degradative enzymes (several distinct DNases, a cysteine protease, NADase, hyaluronidase), and many different secreted toxins (streptolysin S, streptolysin O, streptococcal super antigen, streptokinase) [6].

Although Streptococcus pyogenes remains exquisitely sensitive to penicillin and other beta lactam antibiotics, macrolide resistance has been found in as high as $70 \%$ of Streptococcus pyogenes in Japan [1]. But resistance to lincosamide has also been reported sporadically over the last 20 years [7]. Although cross-resistance to macrolide and lincosamide antibiotics has been observed among a variety of gram-positive bacteria, this multipleresistance phenomenon has not commonly occurred among Streptococcus pyogenes before [7].

The present study was conducted to find out the recent clinical characteristics of Streptococcus pyogene sisolates from respiratory tract at general hospital in the central of Japan.

\section{Materials and Methods}

\subsection{Strains and Clinical Data Collection}

A total of 161 Streptococcus pyogenes was obtained from various clinical specimens at Daido Hospital in 2014. Daido Hospital is a 404-bed private general hospital in the central region of Japan. We used medical records appended to clinical species for the analysis of clinical feature at Daido Hospital. We considered several isolates from the same region of the same patient as one isolate per one patient for the analysis in this study. All streptococcus isolates were identified by standard conventional biochemical methods or the VITEK2 system (bioMérieux, Durham NC, USA). Our experimental design was approved by the ethics committee at Daido hospital.

\subsection{Antimicrobial Susceptibility Analysis}

Streptococcus pyogenes isolates were examined for 15 antibiotic susceptibilities as follows S/ABPC; sulbactam/ampicillin, PIPC; piperacillin, AMPC; amoxicillin, CTM; cefotiam, FMOX; flomoxef, CAZ; ceftazidime, CTRX; ceftriaxone, VCM; vancomycin, FOM; fosfomycin, PAPM; panipenem, MEPM; meropenem, CAM; clarithromycin, CLDM; clindamycin, MINO; minocycline, CPFX; ciprofloxacin. Minimal inhibitory concentrations (MICs) were determined at clinical laboratory in Daido Hospital using broth micro dilution methodology with the VITEK2 system. Evaluation of susceptibilities was calculated based on Clinical Laboratory Standard Institute (CLSI) break point [8].

\subsection{Statistical Analysis of the Data}

We conducted the statistical analysis with the chi-squared test or Fisher's exact test when appropriate. Differences were considered significant when $p$ was $<0.05$.

\section{Results}

First of all, we confirmed that all Streptococcus pyogenes isolates were beta hemolysis in this study. Next, we compared the differences between respiratory tract and no respiratory tract isolates in this study. Respiratory tract isolates were defined as isolation from pharynx, nasal discharge, tonsillar, and sputum. Table 1 demon- 
Table 1. Clinical characteristic of Streptococcus pyogenes isolates.

\begin{tabular}{|c|c|c|c|}
\hline & Respiratory tract $(\mathrm{n}=136)$ & No respiratory tract $(\mathrm{n}=25)$ & $p$ value \\
\hline \multicolumn{4}{|l|}{ Gender } \\
\hline Male & 90 & 12 & 0.08 \\
\hline Female & 46 & 13 & \\
\hline \multicolumn{4}{|l|}{ Age } \\
\hline $0-1$ & 6 & 2 & 0.80 \\
\hline $1-18$ & 122 & 9 & $<0.01$ \\
\hline $19-64$ & 5 & 12 & $<0.01$ \\
\hline $65-$ & 3 & 2 & 0.36 \\
\hline \multicolumn{4}{|l|}{ Season } \\
\hline Spring & 42 & 7 & 0.77 \\
\hline Summer & 31 & 8 & 0.32 \\
\hline Fall & 28 & 4 & 0.80 \\
\hline Winter & 35 & 6 & 0.85 \\
\hline \multicolumn{4}{|l|}{ Antomicrobial susceptibility } \\
\hline Minocycline susceptible & 111 & 21 & 1.00 \\
\hline Minocycline no susceptible & 25 & 4 & \\
\hline Clarithromycin susceptible & 45 & 17 & $<0.01$ \\
\hline Clarithromycin no susceptible & 91 & 8 & \\
\hline Clindamycin susceptible & 98 & 21 & 0.32 \\
\hline Clindamycin no susceptible & 38 & 4 & \\
\hline Ciprofloxacin susceptible & 90 & 16 & 0.83 \\
\hline Ciprofloxacin no susceptible & 46 & 9 & \\
\hline
\end{tabular}

strated the comparative analysis of Streptococcus pyogenes between respiratory tract and no respiratory tract. One hundred sixty-one respiratory tract-associated Streptococcus pyogenes were isolated among which 102 (63.3\%) were from male and 59 (36.7\%) were from female. There was no significant difference of gender between respiratory tract and no respiratory tract isolates. The age incidence among $0-1$ years age group was 8 (5\%) [respiratory tract-6, no respiratory tract-2], among1-18 years age group, 131 (81.3\%) [respiratory tract-122, no respiratory tract-9] ( $p<0.01)$, among 19 - 64 years age group, $17(10.6 \%)$ [respiratory tract-5, no respiratory tract-12] ( $p<0.01)$, and in 65-years it was $5(3.1 \%)$ [respiratory tract-3, no respiratory tract-2]

Next, we investigated the relationship between season and bacterial isolation. However, we did not find any significant differences between respiratory tract and no respiratory tract isolation.

The results of antimicrobial susceptible patterns of Streptococcus pyogenes isolates to four antibiotics tested in this study were shown in Table 1. Because other antibiotics were the most active antibiotics with $100 \%$ susceptible rates. Clarithromycin was less antimicrobial effective in respiratory tract disease than in no respiratory tract disease significantly $(p<0.01)$. There were no significant differences of other three antibiotics between respiratory tract and no respiratory tract isolation. Furthermore, we compared the differences among respiratory tract isolations in this study. We categorized four origins as follows; pharynx, nasal discharge, tonsillar, and sputum. Table 2 demonstrated the comparative analysis of Streptococcus pyogenes among respiratory tract. There was no significant difference of gender among respiratory tract isolates. However, there was significant differences of age among respiratory tract $(p<0.05)$. Furthermore, the numbers from nasal discharge were 
Table 2. Clinical characteristic of Streptococcus pyogenes isolates from various respiratory tract.

\begin{tabular}{|c|c|c|c|c|c|}
\hline & Nasal discharge $(\mathrm{n}=56)$ & Pharynx $(\mathrm{n}=35)$ & Tonsillar $(\mathrm{n}=39)$ & Sputum $(n=6)$ & $p$ value \\
\hline \multicolumn{6}{|l|}{ Gender } \\
\hline Male & 37 & 25 & 25 & 3 & 0.75 \\
\hline Female & 19 & 10 & 14 & 3 & \\
\hline \multicolumn{6}{|l|}{ Age } \\
\hline $0-1$ & 6 & 0 & 0 & 0 & 0.03 \\
\hline $1-18$ & 50 & 33 & 38 & 1 & $<0.01$ \\
\hline $19-64$ & 0 & 2 & 1 & 2 & $<0.01$ \\
\hline $65-$ & 0 & 0 & 0 & 3 & $<0.01$ \\
\hline \multicolumn{6}{|l|}{ Season } \\
\hline Spring & 19 & 5 & 14 & 4 & 0.03 \\
\hline Summer & 11 & 8 & 11 & 1 & 0.78 \\
\hline Fall & 14 & 8 & 6 & 0 & 0.40 \\
\hline Winter & 12 & 14 & 8 & 1 & 0.17 \\
\hline \multicolumn{6}{|l|}{ Antomicrobial susceptibility } \\
\hline Minocycline susceptible & 42 & 29 & 34 & 6 & 0.28 \\
\hline Minocycline no susceptible & 14 & 6 & 5 & 0 & \\
\hline Clarithromycin susceptible & 13 & 14 & 15 & 3 & 0.16 \\
\hline Clarithromycin no susceptible & 43 & 21 & 24 & 3 & \\
\hline Clindamycin susceptible & 38 & 25 & 30 & 5 & 0.72 \\
\hline Clindamycin no susceptible & 18 & 10 & 9 & 1 & \\
\hline Ciprofloxacin susceptible & 40 & 23 & 23 & 4 & 0.66 \\
\hline Ciprofloxacin no susceptible & 16 & 12 & 16 & 2 & \\
\hline
\end{tabular}

significantly higher than any other sites $(p<0.01)$. Sputum among respiratory tract isolation was most isolated from spring significantly $(p=0.03)$. From antimicrobial resistance, we did not find any significant differences of among respiratory tract isolation.

\section{Discussion}

In this study, we described the characteristics of respiratory tract associated Streptococcus pyogenes isolates in 2014 at general hospital in the central of Japan. We found the numbers of bacteria from respiratory tract were about five times as same as those from no respiratory tract.

With respect to gender, the numbers of respiratory tract associated Streptococcus pyogenes from male tended to be greater than that from female. But we did not find significant result of gender.

We clarified Streptococcus pyogenes with age distribution. The present study reveals that the numbers of Streptococcus pyogenes isolated from child between 1 and 18were most popular. However, the numbers of no respiratory tract associated Streptococcus pyogenes isolated from adult between 19 and 64 were most popular. In the analysis of season, we did not find any significant differences between respiratory tract and no respiratory tract. Previous report showed that the lowest carriage rates of Streptococcus pyogenes are in adults, whereas children living in crowded conditions in temperature climates during the winter months are highly affected.

In the analysis of antimicrobial susceptibility, we did not find any penicillin and cephalosporin-resistant Streptococcus pyogenes in our study. But we demonstrated that clarithromycin was less antimicrobial effective 
in respiratory tract disease than in no respiratory tract disease significantly. Macrolide including clarithromycin is popular for treatment of respiratory infection disease in Japan [9]. Furthermore, low-dose macrolide therapy is also popular for treatment of chronic rhinosinusitis and chronic respiratory failure disease [10] [11]. Our result suggested that inadequate usage of macrolide may increase the macrolide resistant bacteria. The differences of clarithromycin antimicrobial susceptibility pattern also indicated that no respiratory tract-associated Streptococcus pyogenes were not spread from respiratory tract-associated Streptococci. The clarithromycin-resistant gene (phage-associated gene) is not easily deleted generally [12].

Further investigation revealed that the numbers of Streptococcus pyogenes from nose were most popular among respiratory tract. This result supported that Streptococcus pyogenes was air-boned pathogen and it transmitted from nose firstly [13]. Our result also presented that the numbers of Streptococcus pyogenes from upper respiratory tract to lower respiratory tract were decreasing.

Although we did not find significant differences of gender, and antimicrobial susceptibility among four respiratory tract sites, our result may imply several tendency. The numbers isolated from male were higher than those isolated from female. Clarithromycin resistant rate isolated from nose was higher than any other sites. Further epidemiological analysis is needed for the clarification of several problems about respiratory tract-associated Streptococcus pyogenes.

\section{Conclusion}

Although several antibiotics such as penicillin are effective against Streptococcus pyogenes, incidence of Streptococcus pyogenes infection is not decreasing worldwide. Severe invasive Streptococcus pyogenes disease such as streptococcus toxic shock syndrome may occur by dissemination to other organs of the body if not treated adequately. Our investigation aims are not only to reduce the morbidity and mortality in the patients but also to control the emergence and spread of resistance among Streptococcus pyogenes. The results from our study strongly suggest the need for continuous epidemiological surveillance of antibiotic resistant pathogen such as macrolide-resistant Streptococcus pyogenes isolated from respiratory tract.

\section{Acknowledgements}

We thank Mr. Masashi Ishihara and Ms. Miwako Fujimura for special encouragement. This study was supported by a grant-in-aid for research from the Nagoya City University, Japan.

\section{References}

[1] Tart, A.H., Walker, M.J. and Musser, J.M. (2007) New Understanding of the Group A Streptococcus Pathogenesis Cycle. Trends. Microbiol., 15, 318-325. http://dx.doi.org/10.1016/j.tim.2007.05.001

[2] Stevens, D.L. (2000). Group A Beta-Hemolytic Streptococci: Virulence Factors, Pathogenesis, and Spectrum of Clinical Infections. In: Stevens, D.L. and Kaplan, E.L., Eds., Streptococcal Infections, Oxford University Press, New York, 19-36.

[3] Gawaorzewska, E. and Colman, G. (1988) Changes in the Patterns of Infection Caused by Streptococcus pyogenes. Epidemiol Infect., 100, 257-269. http://dx.doi.org/10.1017/S095026880006739X

[4] Anjos, L.M., Marcondes, M.B., Lima, M.F., Mondelli, A.L. and Okoshi, M.P. (2014) Streptococcal Acute Pharyngitis. Rev Soc Bras Med Trop., 47, 409-413. http://dx.doi.org/10.1590/0037-8682-0265-2013

[5] Cho, K.H. and Caparon, M. (2006) Genetic of Group A Streptococci. In: Fischetti, V.A., Novick, R.P., Ferretti, J.J., Portnoy, D.A. and Rood, J.I., Eds., Gram Positive Pathogens, ASM Press, Washington DC, 59-73.

[6] Tan, L.K., Eccersley, L.R. and Sriskandan, S. (2014) Current Views of Haemolytic Streptococcal Pathogenesis. Curr Opin Infect Dis., 27, 155-164. http://dx.doi.org/10.1097/QCO.0000000000000047

[7] Wajima, T., Chiba, N., Morozumi, M., Shouji, M., Sunaoshi, K., Sugita, K., Tajima, T. and Ubukata, K., GAS Surveillance Study Group (2014) Prevalence of Macrolide Resistance among Group A Streptococci Isolated from Pharyngotonsillitis. Microb Drug Resist., 20, 431-435. http://dx.doi.org/10.1089/mdr.2013.0213

[8] Clinical and Laboratory Standards Institute (CLSI) (2014) Performance Standards for Antimicrobial Susceptibility Testing: 24th Informational Supplement, Clinical and Laboratory Standards Institute M100-S24, Wayne.

[9] Goto, H., Shimada, K., Ikemoto, H. and Oguri, T., Study Group on Antimicrobial Susceptibility of Pathogens Isolated from Respiratory Infections (2009)Antimicrobial Susceptibility of Pathogens Isolated from More than 10,000 Patients with Infectious Respiratory Diseases: A 25-Year Longitudinal Study. J Infect Chemother., 15, 347-360. 
http://dx.doi.org/10.1007/s10156-009-0719-3

[10] Cervin, A. and Wallwork, B. (2007) Macrolide Therapy of Chronic Rhinosinusitis. Rhinology, 45, 259-267.

[11] Kudoh, S. and Keicho, N. (2012) Diffuse Panbronchiolitis. Clin Chest Med., 33, 297-305. http://dx.doi.org/10.1016/j.ccm.2012.02.005

[12] Michos, A.G., Bakoula, C.G., Braoudaki, M., Koutouzi, F.I., Roma, E.S., Pangalis, A., Nikolopoulou, G., Kirikou, E. and Syriopoulou, V.P. (2009) Macrolide Resistance in Streptococcus pyogenes: Prevalence, Resistance Determinants, and EMM Types. Diagn Microbiol Infect Dis., 64, 295-299. http://dx.doi.org/10.1016/j.diagmicrobio.2009.03.004

[13] Sherertz, R.J., Bassetti, S. and Bassetti-Wyss, B. (2001) “Cloud” Health-Care Workers. Emerg Infect Dis., 7, $241-244$. http://dx.doi.org/10.3201/eid0702.010218 